\title{
Effect of Bronchoscopist-Directed Sedation and Other Factors on Patient Comfort during Diagnostic Flexible Bronchoscopy
}

\author{
Rahul Magazine $\mathbb{D}^{1},{ }^{1}$ Keerthi Nedumala Sisupalan, ${ }^{1}$ Vyshak Uddur Surendra, ${ }^{1}$ \\ Bharti Chogtu $\mathbb{D}^{2},{ }^{2}$ Preetam Rajgopal Acharya $\mathbb{D}^{3},{ }^{3}$ and Vasudeva Guddattu ${ }^{4}{ }^{4}$ \\ ${ }^{1}$ Department of Respiratory Medicine, Kasturba Medical College, Manipal Academy of Higher Education, Manipal, \\ Karnataka 576104, India \\ ${ }^{2}$ Department of Pharmacology, Kasturba Medical College, Manipal Academy of Higher Education, Manipal, \\ Karnataka 576104, India \\ ${ }^{3}$ Department of Respiratory Medicine, Kasturba Medical College, Manipal Academy of Higher Education, Mangalore, \\ Karnataka, India \\ ${ }^{4}$ Department of Data Science, Prasanna School of Public Health, Manipal Academy of Higher Education, Manipal, \\ Karnataka 576104, India \\ Correspondence should be addressed to Bharti Chogtu; bhartimagazine@gmail.com
}

Received 3 May 2021; Revised 22 December 2021; Accepted 23 December 2021; Published 21 January 2022

Academic Editor: Abdel Halim Salem

Copyright (c) 2022 Rahul Magazine et al. This is an open access article distributed under the Creative Commons Attribution License, which permits unrestricted use, distribution, and reproduction in any medium, provided the original work is properly cited.

\begin{abstract}
Background. The factors affecting patient comfort during flexible bronchoscopy are not sufficiently addressed in resource-limited countries, and a need to devise strategies to improve patient experience is felt. The present study was undertaken to assess the effect of sedation and other factors on patient comfort during flexible bronchoscopy. Methods. A total of 374 patients, aged $\geq 18$ years undergoing flexible bronchoscopy, were enrolled in this prospective, single-center, cross-sectional study. The baseline clinical data of sedation and nonsedation groups were recorded. Anxiety level was assessed using Visual Analog Scale (VAS). Postprocedure VAS score (primary outcome measure) assessed the discomfort related to flexible bronchoscopy. Patient-reported willingness for a repeat procedure and occurrence of adverse events were used as secondary outcome measures. Based on the median of VAS score, the study population was divided into low discomfort and high discomfort groups, and the factors affecting comfort levels in these two groups were noted. Results. Postprocedural VAS score (median (Q1, Q3)) for sedation and nonsedation groups was $20(15,30)$ and $25(20$, $40)$, respectively $(<0.001)$. Willingness for a repeat procedure was significantly more in the sedation group $(p=0.002)$. In the low and high discomfort groups, the preprocedural anxiety level (median (Q1, Q3)) was $20(10,25)$ and $30(20,40)$, respectively $(p<0.001)$. Conclusion. Bronchoscopist-directed sedation significantly reduces patient discomfort and increases their willingness for a repeat procedure. Midazolam-fentanyl combination and lower prebronchoscopy anxiety are associated with lower discomfort levels during flexible bronchoscopy. This study is registered with the Clinical Trial Registry of India (CTRI/2018/11/016328).
\end{abstract}

\section{Introduction}

Flexible bronchoscopy, a commonly performed procedure in a pulmonary medicine unit, provides vital input by allowing direct observation of the airway and acquiring biological material for a comprehensive evaluation of the patients' clinical condition. The use of sedation during this procedure is increasingly becoming the standard of care due to the expectations of the patient to experience a comfortable procedure $[1,2]$. Having said that, the majority $(59.4 \%)$ of bronchoscopists in developing countries do not use sedation during flexible bronchoscopy, which contrasts with the Western world where $4-10 \%$ routinely use sedation to enhance patient comfort $[3,4]$. There could be multiple reasons, such as lack of experience in use of sedatives, fear of complications arising due to sedation, and so on, for such underutilization of sedation during flexible bronchoscopy $[3,5]$. Although sedatives have a major impact on the level of comfort during flexible bronchoscopy, there are other factors that can influence it as well [5-7]. Being aware of these 
factors can help the bronchoscopist to devise interventions to address these more effectively, thus improving the comfort level of the patient during flexible bronchoscopy. There is a pressing need to generate data from our country regarding the benefits and risks of bronchoscopist-directed conscious sedation and to understand the factors which impact patients' level of comfort during flexible bronchoscopy. With this background, the present study was conducted to assess the effect of bronchoscopist-directed conscious sedation on patient comfort during flexible bronchoscopy and to study the factors affecting patient comfort during flexible bronchoscopy.

\section{Materials and Methods}

2.1. Setting. A prospective, single-center, cross-sectional study was conducted at a tertiary care teaching hospital in South India after obtaining approval from the institutional ethics committee. After taking the informed consent, a total of 374 patients, who met the inclusion criteria for the study, were enrolled from December 2018 to October 2020. Consecutive eligible study subjects were allocated to one of the two groups, the sedation group or nonsedation group, based on whether they accepted bronchoscopist-directed sedation during flexible bronchoscopy or not (Figure 1). Written informed consent was obtained from the study participants after they read the patient information sheet about the study, which had been provided to them. Inclusion criterion included all patients aged $\geq 18$ years undergoing flexible bronchoscopy in the Department of Respiratory Medicine. Patients who had undergone flexible bronchoscopy previously, patients on mechanical ventilation, patients who had a tracheostomy in place, patients having psychiatric disorders, and patients with any difficulty in communication that compromised their ability to answer questions were excluded from the study.

A prebronchoscopy questionnaire was administered to note the baseline clinical data and patients were asked to rate their anxiety level before flexible bronchoscopy using Visual Analog Scale (VAS). This was completed 12 hours prior to flexible bronchoscopy for all enrolled patients.

VAS is a psychometric response scale, which measures the patients' subjective feeling, and it was used to assess patients' anxiety prior to flexible bronchoscopy on a $10 \mathrm{~cm}$ horizontal line [7]. A good correlation has been noted between patients' VAS and blinded observer's VAS [8]. The left end $(0 \mathrm{~cm})$ of the horizontal line indicates "no anxiety," and the right end $(10 \mathrm{~cm})$ indicating "severe anxiety." The patients were asked to mark on a $10 \mathrm{~cm}$ horizontal line at the point corresponding to their subjective degree of anxiety before flexible bronchoscopy. VAS score was determined by measuring the distance from the left end to the mark made by the patients.

During the procedure, all patients were in the supine position. Supplemental oxygen was administered to all patients. The oxygen was titrated to keep (oxygen saturation) $\mathrm{SpO}_{2} \geq 95 \%$. Bronchoscopist-directed conscious sedation was administered if the patient had indicated a prior willingness for its use; otherwise, flexible bronchoscopy was

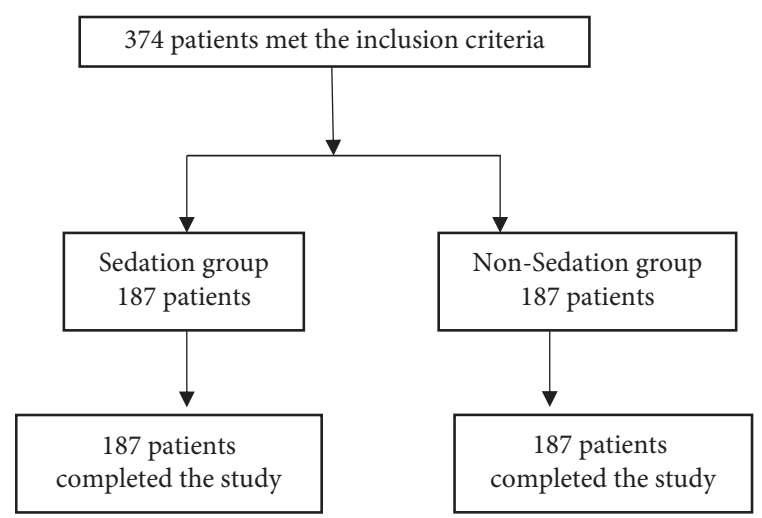

FIgURE 1: Consort diagram showing patient allocation to two groups.

done without sedation. The sedatives used were chosen by the bronchoscopists, and their dosage titrated based on the patients' clinical parameters-their comfort level and vitals-during the procedure. After local application of lignocaine, the flexible bronchoscopy was inserted through the nostril to reach the oropharynx. If the nasal route was not technically feasible, then the oral route was opted. Two $\mathrm{ml}$ of topical $2 \%$ lignocaine was sprayed on the vocal cords through the flexible bronchoscopy.

One minute later, the bronchoscope was negotiated across the vocal cords, one $\mathrm{ml}$ of topical $2 \%$ lignocaine was sprayed over the trachea, and one $\mathrm{ml}$ each into the right and left main bronchi near the carina. Once the cough subsided, the flexible bronchoscope was advanced into both bronchi for the examination of the entire bronchial tree and for carrying out the sampling procedure planned for the patient. If the patient developed a recurrent cough, an additional $2 \%$ lignocaine spray was used in aliquots of one $\mathrm{ml}$ at a time. Duration of the procedure was noted from the time of scope insertion to the time of removal of scope. The total lignocaine dose used for the procedure was also recorded.

After the procedure (2 hours later), again a $10 \mathrm{~cm}$ Visual Analogue Scale (VAS) was used to assess the patients' subjective degree of discomfort during flexible bronchoscopy. After 8 hours, patients were asked about their willingness to return for a repeat procedure if needed. For this, the patients were asked to choose from the following five options: absolutely no, probably no, I do not know, probably yes, or absolutely yes.

2.2. Outcome Measures. Patient-marked VAS score for assessing discomfort related to flexible bronchoscopy was used as a primary outcome measure. The discomfort felt by patients in the sedation and nonsedation groups were assessed using this score. The median of the scores of the whole study population $(N=374)$ was used to divide it into low discomfort and high discomfort groups [7]. The factors that affected comfort levels were noted based on this division. Patient-reported willingness for a repeat procedure and occurrence of adverse events in sedation and nonsedation groups were used as secondary outcome measures. 
2.3. Statistical Analysis. The SPSS version 25.0 (SPSS Inc., Chicago, IL, USA) was used for statistical analysis. Frequency and percentage were used to summarize categorical variables. Mean and standard deviation were used to summarize normally distributed continuous variables. Median and interquartile range was used to summarize postbronchoscopy VAS score. Chi-square test was used for finding the association between two categorical variables, and independent $t$-test was used to compare the mean of continuous variables across two groups (sedated vs. nonsedated, high discomfort vs. low discomfort). Man$\mathrm{n}$-Whitney $U$-test was used to compare median of nonnormally distributed variables across two groups. $p$ value of $<0.05$ was considered as statistically significant.

2.4. Sample Size estimation. For a power of $80 \%$ at $95 \%$ confidence interval, a minimum of 187 patients, each in the sedation and nonsedation group, were recruited for the study.

\section{Results}

A total of 374 patients were included in the study, with 187 patients each in the sedation and nonsedation group. The baseline characteristics of each group are summarized in Table 1.

The mean duration of the procedure in the sedation and nonsedation group was $14.25 \pm 5.43$ and $13.87 \pm 5.12$ minutes $(p=0.487)$. The lignocaine dose (mean $\pm \mathrm{SD})$ used was $185.88 \mathrm{mg} \pm 12.68$ and $191.98 \mathrm{mg} \pm 19.94$, respectively $(p<0.001)$. Postprocedural discomfort score and patientreported willingness for a repeat procedure in the sedation and nonsedation group are shown in Table 2 .

In the sedation group, the adverse events were as follows: uneventful (146 (78.1\%)), hemoptysis (16 (8.6\%)), desaturations not requiring interventions/termination of procedure $(19(10.2 \%))$, desaturations requiring interventions (2 $(1.1 \%))$, tachycardia (2 (1.1\%)), hypotension (0), bronchospasm $(1(0.5 \%))$, and bleeding $(1(0.5 \%))$. In the nonsedation group, the adverse events were as follows: uneventful (158 (84.5)), hemoptysis (14(7.5\%)), desaturations not requiring interventions/termination of procedure $(8(4.3 \%))$, desaturations requiring interventions $(2(1.1 \%))$, tachycardia $(2(1.1 \%))$, hypotension $(1(0.5 \%))$, bronchospasm (0), and bleeding (2(1.1\%)). There was no significant difference between the two groups so far as the occurrence of adverse events was concerned $(p=0.386)$.

The median VAS score of the level of discomfort related to flexible bronchoscopy was $25 \mathrm{~mm}$. The patients were divided into two groups based on this median of the VAS score: low discomfort $(<25$ VAS) and high discomfort $(\geq 25$ VAS). Out of a total of 374 cases, 170 patients were in the low discomfort group and 204 patients in the high discomfort group. Factors affecting patient comfort during flexible bronchoscopy are summarized in Table 3. Factors affecting patient comfort in the sedation and nonsedation groups during flexible bronchoscopy are summarized in Table 4 . The median of the preprocedure anxiety VAS score was 23 .
Patients were divided into low anxiety and high anxiety levels based on the median for the preprocedure anxiety. The mean age in years $( \pm \mathrm{SD})$ in the low anxiety and high anxiety groups was $51.13 \pm 14.38$ and $54.77 \pm 13.78(p=0.013)$.

\section{Discussion}

The introduction of newer technologies and techniques has enhanced the utility of diagnostic flexible bronchoscopy but at the same time has made it a much more complex and time-consuming procedure than before. This can have adverse consequences on both the patients' comfort level as well as bronchoscopists' ease of doing the procedure. The use of conscious sedation during flexible bronchoscopy is mainly directed at improving these two aspects of the procedure; in addition, it can also have a favorable impact on the bronchoscopy-induced sympathetic response [5, 9]. Although enhancing patient comfort during flexible bronchoscopy is a need that all bronchoscopists agree with, yet there is a debate on whether patient comfort should be given precedence or the successful completion of the procedure should be the primary goal [10]. The debate may rage on, but the fact of the matter is that both these aspects need to be taken care of simultaneously. Optimizing the factors of which sedation is an important one that affect patient comfort has the potential to create favorable conditions for successful completion of the procedure as well. The assessment of patient's comfort after the completion of flexible bronchoscopy can help in improving the protocols, which bronchoscopists adopt for mitigating discomfort during future procedures. Even guidelines suggest that along with the diagnostic usefulness of flexible bronchoscopy, patient's comfort and satisfaction is an important outcome measure of the procedure [1].

Inadequate sedation is known to increase patient discomfort and reduce the willingness of the patient to return for a repeat procedure $[11,12]$. In our study also, we found that postprocedure VAS score was significantly higher, indicating more discomfort, in nonsedation group when compared to the sedation group. In the flexible bronchoscopy and other clinical settings as well, the patient satisfaction determines patients' willingness to return; thus, willingness to return has been used as a surrogate indicator of patient comfort $[2,13]$. The willingness to return for repeat flexible bronchoscopy varies greatly ( $25.1 \%$ to $93 \%)$ in different studies $[2,12,14]$. Higher percentages were noted in studies conducted in developed countries like Japan and the United States of America, whereas lower percentages were reported from developing countries like Egypt. In our study, only $29.6 \%$ in the sedation group were willing for a repeat procedure. In spite of this lower percentage of willingness for a repeat procedure in our sedation group, bronchoscopist-directed conscious sedation did increase the willingness when compared to the nonsedation group (19.3\%). In developed countries, more robust protocols aimed at patient's comfort are employed, which could explain the higher percentage of patients willing for a repeat procedure in their studies. Class of sedatives chosen, the number of drugs used, their dosage, and the manner of 
TABLE 1: Demographic characteristics of patients in sedation and nonsedation groups $(n=374)$.

\begin{tabular}{|c|c|c|c|}
\hline Characteristics & Sedation $(n=187)$ & Nonsedation $(n=187)$ & $p$-value \\
\hline Age (years) $($ mean \pm SD) & $51.64 \pm 14.06$ & $54.29 \pm 14.23$ & $0.071^{*}$ \\
\hline Male/female & $107 / 80$ & $115 / 72$ & $0.40^{\#}$ \\
\hline Weight in $\mathrm{kg}($ mean $\pm \mathrm{SD})$ & $52.71 \pm 11.06$ & $51.84 \pm 9.57$ & $0.418^{*}$ \\
\hline Height in $\mathrm{cm}($ mean $\pm \mathrm{SD})$ & $158.06 \pm 6.1$ & $158.57 \pm 6.28$ & $0.481^{*}$ \\
\hline $\mathrm{BMI} \mathrm{kg} / \mathrm{m}^{2}($ mean $\pm \mathrm{SD})$ & $21.02 \pm 3.83$ & $20.58 \pm 3.28$ & $0.239^{*}$ \\
\hline Comorbidities, $N(\%)$ & $126(67.0)$ & $117(62.6)$ & $0.329^{\#}$ \\
\hline \multicolumn{4}{|l|}{ Indication for bronchoscopy } \\
\hline Tubercular infection & $75(40.1)$ & $73(39.0)$ & \multirow{3}{*}{$0.973^{\#}$} \\
\hline Nontubercular infection & $69(36.9)$ & $71(38.0)$ & \\
\hline Malignancy & $43(23.0)$ & $43(23.0)$ & \\
\hline Preprocedural anxiety level (VAS score) (median (Q1, Q3) & $20(15,35)$ & $25(15,35)$ & $0.996^{* *}$ \\
\hline \multicolumn{4}{|l|}{ Route of bronchoscope insertion } \\
\hline Transnasal & $173(92.5)$ & $168(89.8)$ & \multirow{2}{*}{$0.831^{\#}$} \\
\hline Transoral & $14(7.5)$ & $19(10.2)$ & \\
\hline \multicolumn{4}{|l|}{ Bronchoscopic procedure performed } \\
\hline Bronchial lavage & $187(50 \%)$ & $187(50 \%)$ & - \\
\hline Biopsy/TBNA & $24(12.8 \%)$ & $19(10.2 \%)$ & $0.418^{\#}$ \\
\hline Bronchial brushing & $40(21.4 \%)$ & $47(24.6 \%)$ & $0.461^{\#}$ \\
\hline
\end{tabular}

${ }^{\#}$ Chi-square test. ${ }^{*}$ Independent sample $t$-test. ${ }^{* *}$ Mann-Whitney $U$ test. TBNA: transbronchial needle aspiration.

Table 2: Postflexible bronchoscopy outcome measures.

\begin{tabular}{|c|c|c|c|}
\hline Outcome measures & Sedation $(n=187)$ & Nonsedation $(n=187)$ & $p$-value \\
\hline Postprocedural discomfort score (VAS score) (median (Q1, Q3) & $20(15,30)$ & $25(20,40)$ & $<0.001^{* *}$ \\
\hline \multicolumn{4}{|c|}{ Patient-reported willingness for a repeat procedure, if required in the future } \\
\hline Absolutely no & $15(8)$ & $37(19.8)$ & \\
\hline Probably no & $33(17.6)$ & $43(23)$ & \\
\hline I do not know & $84(44.9)$ & $71(38)$ & $0.002^{\#}$ \\
\hline Probably yes & $52(27.8)$ & $31(16.6)$ & \\
\hline Absolutely yes & $3(1.6)$ & $5(2.7)$ & \\
\hline
\end{tabular}

${ }^{\#}$ Chi-square test. ${ }^{* *}$ Mann-Whitney $U$ test.

administration over time (fixed dose or titrating doses) can affect the level of sedation and thus influence the patient comfort achieved [15].

In various studies, different sedatives-midazolam, fentanyl, propofol, dexmedetomidine, and so on-have been tried and found to improve patient comfort during flexible bronchoscopy [16-21]. The data from the present study suggest that the midazolam-fentanyl combination has a significant impact on reducing patient discomfort. A similar inference was drawn from a randomized, double-blinded, placebo-controlled Indian study where midazolam alone was compared head to head with midazolam-fentanyl combination. In this study, both midazolam and the midazolam-fentanyl combination was better than placebo, and in addition the midazolamfentanyl combination was better than midazolam alone [16]. However, a survey from India showed that only $26.7 \%$ of those who used sedation preferred midazolamfentanyl combination, whereas $58.3 \%$ preferred midazolam alone. Given the fact that less than half of the bronchoscopists in India use sedation at all, it is obvious that a very small percentage of patients must be receiving adequate sedation using the midazolam-fentanyl combination that is indeed a better option, if no contraindications exist [3]. In contrast to this, in Australia and
New Zealand, a combination of two sedatives were used by $94 \%$, and out of these, $96 \%$ used the midazolamfentanyl combination [18].

The safety profile of various sedatives in common use, when compared with placebo, has been well established, and whatever complications do arise are quite manageable $[16,17,22,23]$. Our study also found that the adverse event profile was comparable in both the sedation and nonsedation groups $(p=0.386)$.

For many patients, the thought of undergoing a diagnostic flexible bronchoscopy can be a cause of high anxiety, and this preprocedure anxiety has been documented to be one of the factors associated with increased patient discomfort during flexible bronchoscopy [24, 25]. In the present study also, we found that there was a significant association between higher preprocedure anxiety and higher discomfort experienced during flexible bronchoscopy. Similar findings were found in various studies, and it has been suggested that preprocedure anxiety levels should be routinely assessed using a questionnaire and appropriate measures, like increased sedation, instituted to tackle the expected increase in discomfort among such patients $[6,7]$. Interestingly, in one study, touch and verbal empathy employed prior to flexible bronchoscopy were found to reduce anxiety in patients with heightened anxiety levels 
TABLE 3: Factors affecting patient comfort during flexible bronchoscopy.

\begin{tabular}{|c|c|c|c|}
\hline Characteristics & $\begin{array}{l}\text { Low discomfort VAS }<25 \text {, } \\
\qquad n=170\end{array}$ & $\begin{array}{l}\text { High discomfort VAS } \geq 25, \\
\qquad n=204\end{array}$ & $p$-value \\
\hline Age (mean years $\pm S D)$ & $52.00 \pm 14.54$ & $53.76 \pm 13.87$ & $0.232^{*}$ \\
\hline Male: female & $108: 62$ & $114: 90$ & $0.134^{\#}$ \\
\hline \multicolumn{4}{|l|}{ Addictions, $n(\%)$} \\
\hline Smokers (including ex-smokers) & $34(20.0)$ & $51(25.0)$ & \multirow{4}{*}{$0.120^{\#}$} \\
\hline Alcoholics & $2(1.2)$ & $6(2.9)$ & \\
\hline Both (smokers and alcoholics) & $8(4.7)$ & $3(1.5)$ & \\
\hline Nil addictions & $126(74.1)$ & $144(70.6)$ & \\
\hline Any comorbidities, $n(\%)$ & $108(63.53)$ & $135(66.2)$ & $0.593^{\#}$ \\
\hline \multicolumn{4}{|l|}{ Socioeconomic class, $n(\%)$} \\
\hline Lower class & $2(1.2)$ & $4(2)$ & \multirow{5}{*}{$0.674^{\#}$} \\
\hline Upper lower class & $36(21.2)$ & $49(24.0)$ & \\
\hline Lower middle class & $97(57.1)$ & $116(56.9)$ & \\
\hline Upper middle class & $34(20.0)$ & $32(15.7)$ & \\
\hline Upper class & $1(0.6)$ & $3(1.5)$ & \\
\hline \multicolumn{4}{|l|}{ Indication for flexible bronchoscopy, $n(\%)$} \\
\hline Tubercular & $69(40.6)$ & $79(38.7)$ & \multirow{3}{*}{$0.865^{\#}$} \\
\hline Other infections & $64(37.6)$ & $76(37.3)$ & \\
\hline Malignancy & $37(21.8)$ & $49(24.0)$ & \\
\hline Preprocedural anxiety level (VAS score) (median (Q1, Q3) & $20(10,25)$ & $30(20,40)$ & $<0.001^{* *}$ \\
\hline \multicolumn{4}{|l|}{ Route of bronchoscope insertion, $n(\%)$} \\
\hline Transnasal & $155(91.2)$ & $186(91.2)$ & \multirow{2}{*}{$1.000^{\#}$} \\
\hline Transoral & $15(8.8)$ & $18(8.8)$ & \\
\hline Midazolam used, $n(\%)$ & $58(34.1)$ & $54(26.5)$ & \multirow{2}{*}{$0.068^{\#}$} \\
\hline Midazolam not used, $n(\%)$ & $112(65.9)$ & $150(73.5)$ & \\
\hline Midazolam fentanyl combination used, $n(\%)$ & $35(20.6)$ & $22(10.8)$ & \multirow{2}{*}{$0.009^{\#}$} \\
\hline Midazolam fentanyl combination not used, $n(\%)$ & $135(79.4)$ & $182(89.2)$ & \\
\hline Lignocaine dose $($ mean $\pm S D)$ & $187.65 \pm 15.55$ & $190.0 \pm 18.03$ & $0.182^{*}$ \\
\hline \multicolumn{4}{|l|}{ Bronchoscopic interventions done, $n(\%)$} \\
\hline Bronchial lavage & $170(100)$ & $204(100)$ & - \\
\hline Biopsy/TBNA & $21(12.4)$ & $22(10.8)$ & $0.636^{\#}$ \\
\hline Bronchial brushing & $41(24.1)$ & $45(22.1)$ & $0.638^{\#}$ \\
\hline \multicolumn{4}{|l|}{ Experience of the operator } \\
\hline Less experienced $(0-4$ years $)$ & $125(73.5)$ & $141(69.1)$ & \multirow{2}{*}{$0.349^{\#}$} \\
\hline More experienced ( $>4$ years) & $45(26.5)$ & $63(30.9)$ & \\
\hline Duration of procedure in minutes $($ mean $\pm S D)$ & $14.10 \pm 4.87$ & $14.02 \pm 5.6$ & $0.884^{*}$ \\
\hline $\begin{array}{l}\text { Procedure-related adverse events during or in the immediate } \\
\text { postprocedure period }\end{array}$ & $35(20.6)$ & $36(17.6)$ & $0.470^{\#}$ \\
\hline
\end{tabular}

${ }^{\#}$ Chi-square test. ${ }^{*}$ Independent Student's $t$-test. ${ }^{* *}$ Mann-Whitney $U$ test.

[26]. On the other hand, providing detailed risk information before flexible bronchoscopy, which is a legal and ethical requirement as well, may result in marginal but significant increase in anxiety levels [27]. In our study, we found higher age to be associated with increased preprocedure anxiety, but since the difference in the mean ages is small, it does not appear to be a clinically significant difference.

While some studies have shown that the experience of the bronchoscopist influences patients' comfort levels, there are others which did not find such an association $[6,7]$. In our study also, we did not note any impact of bronchoscopist experience on patient comfort level. This difference among various studies could be due to the different definitions used for a well-experienced bronchoscopist. In addition, there are subtle variables like the quality of training, which may vary among different training centers, and thus influence patient comfort. The total number of bronchoscopies done by an operator might be a better measure of expertise than a number of years of experience.

Male gender and shorter procedure time have been associated with better patient satisfaction [12]. In our study, the whole study population taken together did not reveal any association of patient discomfort with gender or procedure time, but in the sedation group, male gender was associated with lower discomfort.

4.1. Limitations of the Study. The outcome measure used, namely, the VAS score, is a subjective assessment tool and thus can be influenced by the personal characteristics of the patient. Since randomization was not part of the study design, a more balanced distribution of patients between the sedation and nonsedation groups could have got compromised. It is conceivable that one group could have got more 
TABLE 4: Factors affecting patient comfort in sedation and nonsedation groups during flexible bronchoscopy.

\begin{tabular}{|c|c|c|c|c|c|c|}
\hline \multirow[b]{2}{*}{ Characteristics } & \multicolumn{3}{|c|}{ Sedation $(N=187)$} & \multicolumn{3}{|c|}{ Nonsedation $(N=187)$} \\
\hline & $\begin{array}{l}\text { Low discomfort } \\
\text { VAS }<25, n=103\end{array}$ & $\begin{array}{l}\text { High discomfort } \\
\text { VAS } \geq 25, n=84\end{array}$ & $p$-value & $\begin{array}{l}\text { Low discomfort } \\
\text { VAS }<25, n=67\end{array}$ & $\begin{array}{l}\text { High discomfort } \\
\text { VAS } \geq 25, n=120\end{array}$ & $p$-value \\
\hline Age (mean years $\pm S D)$ & $50.30 \pm 14.05$ & $53.27 \pm 13.98$ & $0.368^{*}$ & $54.61 \pm 15.06$ & $54.11 \pm 13.83$ & $0.637^{*}$ \\
\hline Male: female & $66: 37$ & $41: 43$ & $0.036^{\#}$ & $42: 25$ & $73: 47$ & $0.803^{\#}$ \\
\hline \multicolumn{7}{|l|}{ Addictions, $n(\%)$} \\
\hline Smokers (including ex-smokers) & $17(16.5)$ & $18(21.4)$ & \multirow{3}{*}{$0.303^{\#}$} & $17(25.4)$ & $33(27.5)$ & \multirow{4}{*}{$0.311^{\#}$} \\
\hline Alcoholics & $2(1.9)$ & $2(2.4)$ & & $0(0)$ & $4(3.3)$ & \\
\hline Both (smokers and alcoholics) & $4(3.9)$ & $0(0)$ & & $4(6.0)$ & $3(2.5)$ & \\
\hline Nil addictions & $80(77.7)$ & $64(76.2)$ & & $46(68.7)$ & $80(66.7)$ & \\
\hline Comorbidities, $n(\%)$ & $70(68)$ & $56(66.7)$ & $0.851^{\#}$ & $38(56.7)$ & $79(65.8)$ & $0.217^{\#}$ \\
\hline \multicolumn{7}{|l|}{ Socioeconomic class, $n(\%)$} \\
\hline Lower class & $2(1.9)$ & $0(0)$ & \multirow{5}{*}{$0.480^{\#}$} & $0(0)$ & $4(3.3)$ & \multirow{5}{*}{$0.395^{\#}$} \\
\hline Upper lower class & $20(19.4)$ & $22(26.2)$ & & $16(23.9)$ & $27(22.5)$ & \\
\hline Lower middle class & $55(53.4)$ & $43(51.2)$ & & $42(62.7)$ & $73(60.8)$ & \\
\hline Upper middle class & $25(24.3)$ & $19(22.6)$ & & $9(13.4)$ & $13(10.8)$ & \\
\hline Upper class & $1(1.0)$ & $0(0)$ & & $0(0)$ & $3(2.5)$ & \\
\hline \multicolumn{7}{|c|}{ Indication for flexible bronchoscopy, $n$ (\%) } \\
\hline Tubercular & $39(37.9)$ & $36(42.9)$ & \multirow{3}{*}{$0.475^{\#}$} & $30(44.8)$ & $43(35.8)$ & \multirow{3}{*}{$0.444^{\#}$} \\
\hline Other infections & $42(40.8)$ & $27(32.1)$ & & $22(32.8)$ & $49(40.8)$ & \\
\hline Malignancy & $22(21.4)$ & $21(25.0)$ & & $15(22.4)$ & $28(23.3)$ & \\
\hline Preprocedural anxiety level & & & & & & \\
\hline $\begin{array}{l}\text { before examination (VAS score) } \\
\text { (median }(\mathrm{Q} 1, \mathrm{Q} 3) \text { ) }\end{array}$ & $15(10,20)$ & $35(26,45)$ & $<0.001^{* *}$ & $15(10,20)$ & $35.20(30,45)$ & $<0.001^{* *}$ \\
\hline Lignocaine dose $($ mean $\pm S D)$ & $185.83 \pm 12.41$ & $185.95 \pm 13.09$ & $0.946^{*}$ & $190.45 \pm 19.18$ & $192.83 \pm 20.83$ & $0.392^{*}$ \\
\hline \multicolumn{7}{|c|}{ Bronchoscopic interventions done, $n(\%)$} \\
\hline Bronchial lavage & $103(100)$ & $84(100)$ & - & $67(100)$ & $120(100)$ & - \\
\hline Biopsy/TBNA & $13(12.6)$ & $11(13.1)$ & $0.923^{\#}$ & $8(11.9)$ & $11(9.2)$ & $0.547^{\#}$ \\
\hline Bronchial brushing & $21(20.4)$ & $19(22.6)$ & $0.711^{\#}$ & $20(29.9)$ & $26(21.7)$ & $0.213^{\#}$ \\
\hline $\begin{array}{l}\text { Duration of procedure (minutes) } \\
(\text { mean } \pm S D)\end{array}$ & $13.92 \pm 4.856$ & $14.64 \pm 6.07$ & $0.151^{*}$ & $14.37 \pm 4.93$ & $13.58 \pm 5.22$ & $0.952^{*}$ \\
\hline $\begin{array}{l}\text { Procedure-related adverse events } \\
\text { during or in the immediate } \\
\text { postprocedure period }\end{array}$ & $23(22.3)$ & $18(21.42)$ & $0.417^{\#}$ & $12(17.91)$ & $17(14.16)$ & $0.444^{\#}$ \\
\hline
\end{tabular}

${ }^{\#}$ Chi-square test. ${ }^{*}$ Independent Student's $t$-test. ${ }^{* *}$ Mann-Whitney $U$ test.

such patients who are highly sensitive to even minor discomfort, thus affecting the results. Nevertheless, patient marked VAS is a commonly used tool with its validity backed by a decent amount of evidence, and good correlation noted with a blinded observer marked VAS $[8,28]$. Other factors that can potentially influence patient comfort levels, such as time spent by the bronchoscopist in explaining the procedure, were not assessed. Also, the clinician's satisfaction with the procedure, which has been assessed in similar studies, was not captured [8]. Patient's satisfaction with sedation instrument and clinician's satisfaction with sedation instrument are reliable and validated tools for gastrointestinal endoscopy, and these could be used as well. Despite being potentially useful, these have not been used much in the context of bronchoscopy [29].

\section{Conclusion}

Bronchoscopist-directed conscious sedation significantly reduces the patient's discomfort and increases their willingness for a repeat procedure. Lower prebronchoscopy anxiety levels are associated with lower discomfort levels during flexible bronchoscopy. Midazolam-fentanyl combination is associated with significantly lower levels of discomfort during flexible bronchoscopy. Midazolam used alone also showed a similar trend although not reaching statistical significance.

\section{Data Availability}

The data used to support the findings of this study are available from the corresponding author upon request.

\section{Ethical Approval}

All procedures performed in this study were in accordance with the ethical standards of the institutional and/or national research committee. The study was approved by the institutional ethics committee (IEC: 550/218; dated September 12, 2018).

\section{Consent}

Consent was obtained from the patients. 


\section{Conflicts of Interest}

All Authors declare that they have no conflicts of interest regarding the publication of this paper.

\section{Authors' Contributions}

RM conceived and designed the study, conducted research, provided logistic support, and wrote initial draft and final draft of the paper. KMS conducted research and collected and interpreted data and wrote initial draft. BC conceived and designed the study, organized and interpreted the data, wrote final draft of article, and provided logistic support. PRA wrote and approved final draft. AB and VG analyzed and interpreted the data. All authors have critically reviewed and approved the final draft and are responsible for the content and similarity index of the manuscript.

\section{References}

[1] I. A. Du Rand, J. Blaikley, R. Booton, N. Chaudhuri, V. Gupta, and S. Khalid, "British Thoracic Society guideline for diagnostic flexible bronchoscopy in adults: accredited by NICE," Thorax, vol. 68, pp. i1-i44, 2013.

[2] N. Lechtzin, H. R. Rubin, P. White Jr, M. Jenckes, and G. B. Diette, "Patient satisfaction with bronchoscopy," American Journal of Respiratory and Critical Care Medicine, vol. 166, no. 10, pp. 1326-1331, 2002.

[3] K. Madan, A. Mohan, R. Agarwal, V. Hadda, G. C. Khilnani, and R. Guleria, "A survey of flexible bronchoscopy practices in India: the Indian bronchoscopy survey (2017)," Lung India: Official Organ of Indian Chest Society, vol. 35, pp. 98-107, 2018.

[4] S. Gasparini, "It is time for patients to undergo bronchoscopy without discomfort," European Respiratory Journal, vol. 38, no. 3, pp. 507-509, 2011.

[5] American Society of Anesthesiologists Task Force on Sedation and Analgesia by Non-Anesthesiologists, "Practice guidelines for sedation and analgesia by non-anesthesiologists," Anesthesiology, vol. 96, pp. 1004-1017, 2002.

[6] T. Mitsumune, E. Senoh, and M. Adachi, "Prediction of patient discomfort during fibreoptic bronchoscopy," Respirology, vol. 10, no. 1, pp. 92-96, 2005.

[7] F. Yıldırım, Ş Özkaya, and A. S. Yurdakul, "Factors affecting patients' comfort during fiberoptic bronchoscopy and endobronchial ultrasound," Journal of Pain Research, vol. 29, no. 10 , pp. $775-781,2017$.

[8] M. R. Williams, A. McKeown, F. Dexter et al., "Efficacy outcome measures for procedural sedation clinical trials in adults," Anesthesia \& Analgesia, vol. 122, no. 1, pp. 152-170, 2016.

[9] U. Goneppanavar, R. Magazine, B. Periyadka Janardhana, and S. Krishna Achar, "Intravenous dexmedetomidine provides superior patient comfort and tolerance compared to intravenous midazolam in patients undergoing flexible bronchoscopy," Pulmonary medicine, vol. 2015, Article ID 727530 , 8 pages, 2015.

[10] A. C. Mehta, "Don't lose the forest for the trees," American Journal of Respiratory and Critical Care Medicine, vol. 166, no. 10, pp. 1306-1307, 2002.

[11] M. Contoli, G. Gnesini, D. Artioli et al., "Midazolam in flexible bronchoscopy premedication," Journal of Bronchology \& Interventional Pulmonology, vol. 20, no. 3, pp. 232-240, 2013.
[12] T. Hirose, K. Okuda, H. Ishida et al., "Patient satisfaction with sedation for flexible bronchoscopy," Respirology, vol. 13, no. 5, pp. 722-727, 2008.

[13] B. Sun, J. Adams, E. Orav, D. Rucker, T. Brennan, and H. Burstin, "Determinants of patient satisfaction and willingness to return with emergency care," Annals of Emergency Medicine, vol. 35, no. 5, pp. 426-434, 2000.

[14] A. Madkour, N. Osman, S. Sharkawy, and A. Gomaa, "Assessment of patients' satisfaction with flexible bronchoscopy: initial Egyptian experience," Egyptian Journal of Bronchology, vol. 7, no. 2, pp. 71-76, 2013.

[15] S. De, "Assessment of patient satisfaction and lidocaine requirement during flexible bronchoscopy without sedation," Journal of Bronchology \& Interventional Pulmonology, vol. 16, no. 3, pp. 176-179, 2009.

[16] A. M. Prabhudev, B. Chogtu, and R. Magazine, "Comparison of midazolam with fentanyl-midazolam combination during flexible bronchoscopy: a randomized, double-blind, placebocontrolled study," Indian Journal of Pharmacology, vol. 49, pp. 304-311, 2017.

[17] R. Gonzalez, I. De-La-Rosa-Ramirez, A. Maldonado-Hernandez, and G. Dominguez-Cherit, "Should patients undergoing a bronchoscopy be sedated?" Acta Anaesthesiologica Scandinavica, vol. 47, no. 4, pp. 411-415, 2003.

[18] A. M. Barnett, R. Jones, and G. Simpson, "A survey of bronchoscopy practice in Australia and New Zealand," Journal of Bronchology \& Interventional Pulmonology, vol. 23, no. 1, pp. 22-28, 2016.

[19] J. Hwang, Y. Jeon, H.-P. Park, Y.-J. Lim, and Y.-S. Oh, "Comparison of alfetanil and ketamine in combination with propofol for patient-controlled sedation during fiberoptic bronchoscopy," Acta Anaesthesiologica Scandinavica, vol. 49, no. 9, pp. 1334-1338, 2005.

[20] R. Magazine, S. K. Venkatachala, U. Goneppanavar, V. U. Surendra, V. Guddattu, and B. Chogtu, "Comparison of midazolam and low-dose dexmedetomidine in flexible bronchoscopy: a prospective, randomized, double-blinded study," Indian Journal of Pharmacology, vol. 52, no. 1, pp. 23-30, 2020.

[21] D. Stolz, G. Kurer, A. Meyer et al., "Propofol versus combined sedation in flexible bronchoscopy: a randomised non-inferiority trial," European Respiratory Journal, vol. 34, no. 5, pp. 1024-1030, 2009.

[22] J. P. Pallarés, M. Á. Martínez García, R. L. Reyes, F. S. Moret, and J. L. Sanchís Aldás, "A randomised study of midazolam for sedation in flexible bronchoscopy," Archivos de Bronconeumología, vol. 46, no. 6, pp. 302-309, 2010.

[23] Y. L. Ni, Y. L. Lo, T. Y. Lin, Y. F. Fang, and H. P. Kuo, "Conscious sedation reduces patient discomfort and improves satisfaction in flexible bronchoscopy," Chang Gung Medical Journal, vol. 33, pp. 443-452, 2010.

[24] E. O. Leiten, E. M. H. Martinsen, P. S. Bakke, T. M. L. Eagan, and R. Grønseth, "Complications and discomfort of bronchoscopy: a systematic review," European Clinical Respiratory Journal, vol. 3, no. 1, p. 33324, 2016.

[25] A. Aljohaney, "Level and predictors of anxiety in patients undergoing diagnostic bronchoscopy," Annals of Thoracic Medicine, vol. 14, no. 3, p. 198, 2019.

[26] S. M. Choi, J. Lee, Y. S. Park, C.-H. Lee, S.-M. Lee, and J.-J. Yim, "Effect of verbal empathy and touch on anxiety relief in patients undergoing flexible bronchoscopy: can empathy reduce patients' anxiety?" Respiration, vol. 92, no. 6, pp. 380-388, 2016.

[27] M. Uzbeck, C. Quinn, I. Saleem, P. Cotter, J. J. Gilmartin, and S. T. O'Keeffe, "Randomised controlled trial of the effect of 
standard and detailed risk disclosure prior to bronchoscopy on peri-procedure anxiety and satisfaction," Thorax, vol. 64, no. 3, pp. 224-227, 2009.

[28] M. Jensen, C. Chen, and A. M. Brugger, "Interpretation of visual analog scale ratings and change scores: a reanalysis of two clinical trials of postoperative pain," The Journal of Pain, vol. 4, no. 7, pp. 407-414, 2003.

[29] J. Vargo, K. Howard, J. Petrillo, J. Scott, and D. A. Revicki, "Development and validation of the patient and clinician sedation satisfaction index for colonoscopy and upper endoscopy," Clinical Gastroenterology and Hepatology, vol. 7, no. 2, pp. 156-162, 2009. 